\title{
La biologie des cibles PD- 1 et CTLA-4 et la question des biomarqueurs
}

Marie-Caroline Dieu-Nosjean ${ }^{1}$, Christophe Caux ${ }^{2}$

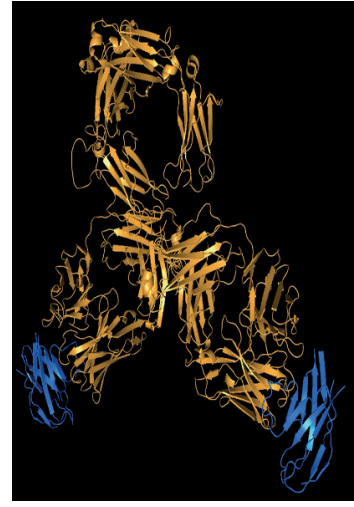

${ }^{1}$ Sorbonne Université UMRS1135, Inserm U1135, Centre d'Immunologie et des Maladies Infectieuses (Cimi-Paris), Faculté de Médecine Sorbonne Université, Paris, France. ${ }^{2}$ UMR Inserm 1052, Centre de Recherche en Cancérologie de Lyon (CRCL), Lyon, France. marie-caroline.dieu-nosjean@ inserm.fr

christophe.caux@lyon.unicancer.fr permettant ainsi d'attaquer avec efficacité les cellules tumorales. La molécule CTLA-4 module l'amplitude de l'activation précoce des LT et inhibe l'activité de CD28, un co-récepteur activateur majeur de ces cellules. La molécule PD-1 est, elle, exprimée par les LT mémoires et effecteurs, et semble intervenir dans la régulation des cellules chroniquement activées, comme lors des processus inflammatoires. Les traitements par anticorps qui découlent de ces découvertes ont pris une place majeure dans l'arsenal des thérapies anti-cancéreuses. Cette revue présente une synthèse des connaissances sur CTLA-4, PD-1 et leurs ligands, de leurs mécanismes d'action et de régulation, ainsi qu'un état des lieux de la compréhension des biomarqueurs associés à la réponse clinique des traitements par anticorps anti-PD-1/PD-Ll et anti-CTLA-4.<

CD28, CTLA-4 (cytolytic T-lymphocyte-associated protein 4) et PD-1 (programmed cell death 1) sont trois récepteurs ayant des implications majeures dans le

${ }^{1}$ CTLA-4 a été découvert par Pierre Golstein au Centre d'Immunologie de MarseilleLuminy (CIML) [1] et voir médecine/sciences, $2019 ; 35: 367-73$.

contrôle de la balance activation/inhibition de la réponse immunitaire, en particulier dans le contrôle de l'activation des lymphocytes T (LT). Nous discuterons ici de la fonction biologique de ces molécules finement régulées et de la puissance de celles-ci comme biomarqueurs chez les patients cancéreux.

\section{De la biologie de la cellule à la biologie des cibles}

Un des enjeux majeurs des stratégies ciblant les points de contrôle immunitaire (en anglais immune checkpoint, ou ICP) est de restaurer les fonctions cytotoxiques des cellules effectrices, fonctions intimement liées à leur état d'activation. Alors que de nombreuses cellules de l'immunité adaptative et innée participent au développement des réponses anti-tumorales, un grand intérêt est actuellement porté à l'activité cytolytique des LT CD8 ${ }^{+}$.

Les mécanismes de contrôle de l'activation du lymphocyte T

\section{L'axe TCR/complexe CMH-peptide}

L'activation complète du LT implique en premier lieu la reconnaissance par son TCR ( $T$-cell receptor) du complexe constitué d'une molécule du complexe majeur d'histocompatibilité (CMH) de classe I ou II et d'un peptide antigénique, présenté par les cellules présentatrices d'antigènes (CPA). Les molécules « accessoires », CD28, à la surface des LT, et ses deux ligands CD80 et CD86, à la surface des CPA, vont ensuite être engagées au niveau de la synapse immunologique pour conduire à l'activation complète du LT. L'engagement du TCR induit la phosphorylation 


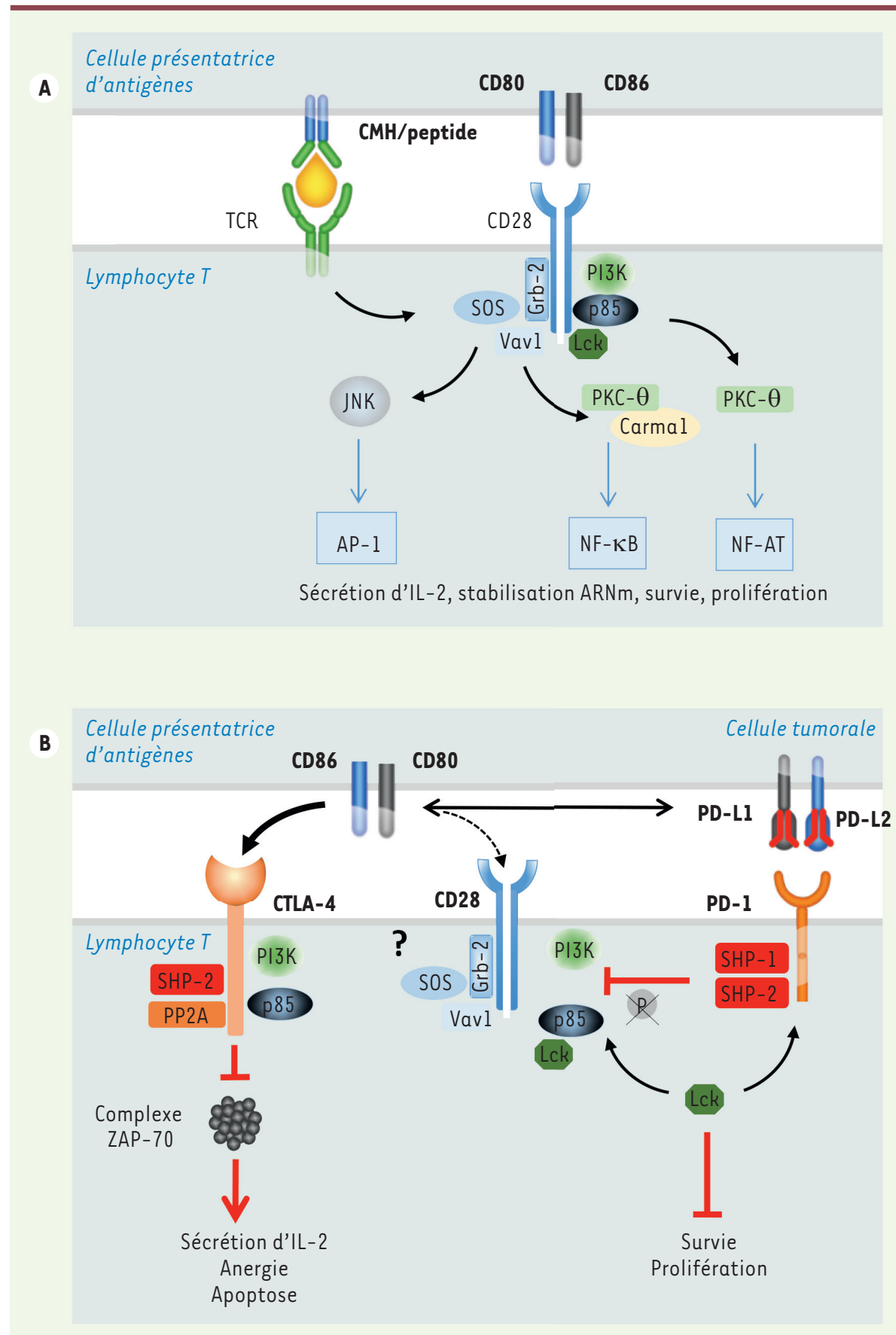

Figure 1. Principaux mécanismes de régulation de l'activation du lymphocyte T dépendante d'un signal TCR. A. Axe TCR/CMH. B. Axe CTLA-4/CD28.

des résidus tyrosines intracellulaires de CD28 par la kinase de la famille des tyrosines kinases Src, Lck (lymphocyte-specific protein tyrosine kinase). Mais, en absence d'activité catalytique intrinsèque dans la région intracellulaire de $\operatorname{CD} 28$, la transduction du signal nécessite le recrutement d'autres molécules, telles que Grb2 (growth factor receptor bound protein 2) et PI3K (phosphoinositide 3-kinase), conduisant in fine à la stabilisation des ARN messagers codant des cytokines et à l'activation des voies NF-AT (nuclear factor of activated T cell), AP-1 (activator protein 1) et NFKB (nuclear factor kappa B) (Figure IA). Cette cascade d'évènements va permettre la sécrétion d'IL(interleukine)-2 et accroître la survie du LT en le préservant, dans un premier temps, de l'épuisement. Cette activation prolongée va cependant aboutir à la mise en place d'un «frein » immunitaire avec l'expression à la membrane du LT de récepteurs régulateurs, tels que CTLA-4 et PD-1, deux molécules découvertes respectivement par Pierre Golstein [1] et Tasuku Honjo [2] ${ }^{2}$.

\section{L'axe CTLA-4/CD28}

La protéine CTLA-4 est stockée constitutivement dans des compartiments intracellulaires du LT au repos, tels que le réseau trans-Golgi, les endosomes et les lysosomes [3, 4]. Dès qu'un certain niveau d'activation est atteint, ces molécules sont rapidement transloquées à la surface de la membrane de la cellule afin d'être fonctionnelles au niveau de la synapse immunologique. C'est ainsi que CTLA-4 va entrer en compétition avec CD28 pour se lier, lui aussi, aux co-récepteurs $C D 80$ et CD86 (Figure 1B). Cependant, son affinité et son avidité étant plus fortes que celles de CD28, CTLA-4 va moduler négativement la signalisation du TCR en recrutant la PI3K et les phosphatases SHP-2 (Src homology region 2 domaincontaining phosphatase-2) et PP2A (protein phosphatase 2A), et ainsi limiter la formation des complexes Zap-70 (zeta-chainassociated protein of $70 \mathrm{kDa}$ ). Cette signalisation va se traduire par une diminution de l'expression de l'IL-2 par le LT, à son anergie, voire à son apoptose. Alors que l'engagement de CTLA-4 sur un LT conventionnel va conduire à la

\footnotetext{
${ }^{2}$ Le comité Nobel a récompensé T. Honjo et J. Allison pour leurs travaux respectifs sur PD-1 et CTLA-4 en immuno-cancérologie (Prix Nobel de Physiologie ou Médecine,
} 2018) (voir médecine/sciences $2019 ; 35: 365-6$ ). 
perte progressive de ses fonctions effectrices, ce même engagement sur un LT régulateur (LTreg) est, quant à lui, indispensable à l'acquisition des fonctions immunosuppressives de ce dernier, en partie via l'activation du promoteur de CTLA-4 par les facteurs de transcription Foxpl et 3 [5-7]. Le mécanisme d'action de l'anticorps thérapeutique anti-CTLA-4, l'ipilimumab, reste néanmoins encore très débattu. Une activité potentiellement déplétante de cet anticorps sur les LTreg a été avancée.

\section{L'axe PD-1/PD-L1-PD-L2}

À la suite de l'engagement du TCR, d'autres récepteurs vont être exprimés à la surface du LT. Parmi ces molécules, PD-l sera, dans un premier temps, un marqueur d'activation. II deviendra un régulateur négatif lorsqu'il aura lié l'un de ses ligands, PD-L1 et PD-L2 (programmed cell death-ligand 1/2), exprimés par des cellules tumorales et immunitaires. Comme CD28, PD-1 va être phosphorylé par Lck (Figure 1B) et les phosphatases SHP-1 et -2 vont ensuite se fixer aux domaines ITIM (immuno-receptor tyrosine-based inhibition motif) et ITSM (immuno-receptor tyrosine-based switch motif) de PD-1. Cette étape est déterminante, puisqu'elle va conduire à la déphosphorylation de CD28, ce qui diminuera fortement la capacité proliférative et les fonctions effectrices du LT [8].

L'efficacité des anticorps anti-PD-1 ou anti-PD-Ll repose ainsi sur la levée de l'inhibition que PD-1 exerce sur CD28. La voie de signalisation de CD28 est alors à nouveau activée, avec une augmentation de la survie, de la prolifération et des fonctions cytotoxiques des LT CD8+ [9]. $\varepsilon$ d'autres termes, le blocage de l'axe PD-1/PD-Ll requière l'expression de CD28 et des molécules de co-stimulation afin de restaurer les fonctions effectrices des LT, comme cela a été démontré dans des modèles d'infection virale chronique et de carcinome colorectal [10]. La molécule de co-stimulation CD80 intervient à un second niveau dans l'activation du LT. En se fixant à PD-Ll, elle devient un puissant compétiteur «naturel » pour PD-1 (Figure 1B); ceci a été démontré par l'administration de molécules solubles de CD80 qui permet de restaurer l'activation des $\mathrm{LT} \mathrm{CD}^{+}{ }^{+}$et CD8+ in vivo, en interférant avec l'axe PD-1/PD-L1 [11].

Le récepteur CD28 est donc assujetti à des régulations extrêmement fines et déterminantes pour les fonctions du LT. Avec sa région extracellulaire, il pourra interagir avec des molécules de co-stimulation et entrer en compétition avec CTLA-4 pour les ligands qu'ils partagent ; avec sa région intra-cytoplasmique, il liera des kinases et des phosphatases, dont SHP-2, associée à PD-1.

\section{Mécanismes de régulation des régulateurs}

PD-1/PD-L1 et de CTLA-4

\section{Co-régulation transcriptionnelle de certains récepteurs inhibiteurs et épuisement des LT}

Une analyse, menée à l'échelle de la cellule unique, a permis de mettre en évidence les mécanismes moléculaires contrôlant l'expression de certains ICP sur les LT CD4 $4^{+}$et CD8+. L'IL-27, une cytokine de la famille de I'IL-12 impliquée dans la différenciation des LTreg producteurs d'IL10 , induit l'expression de deux facteurs de transcription, PRDMl ( $P R$ domain-containing protein 1), ou BLIMP-1 (B-lymphocyte-induced maturation protein-1), et c-MAF (c-musculoaponeurotic fibrosarcoma), qui vont alors activer la transcription d'un ensemble de gènes immuno-régulateurs, dont ceux codant PD-1, TIM-3 (T-cell immunoglobulin and mucindomain containing-3), LAG-3 (lymphocyte-activation gene 3), TIGIT (T cell immunoreceptor with Ig and ITIM domains), et de deux autres récepteurs nouvellement classés comme inhibiteurs, PROCR (protein C receptor) ou EPCR (endothelial protein $C$ receptor) et PDPN (podoplanine) [12]. PROCR promeut l'épuisement total des LT, alors que PDPN limite leur survie aux sites inflammatoires comme l'est par exemple un microenvironnement tumoral.

\section{Dynamique de traduction de certains récepteurs inhibiteurs}

Des travaux récents ont démontré que l'expression de PD-1 est fortement diminuée lorsque des LT CD4 ${ }^{+}, C D 8^{+}$ et $L T \gamma \delta$ circulants activés sont traités par des inhibiteurs des microtubules [13]. Ces drogues conduisent également à la perte d'expression d'autres ICP, tels que TIM-3, LAG-3, TIGIT, CTLA-4 et BTLA ( $B$ - and T-lymphocyte attenuator), alors que l'expression de récepteurs activeurs (0X-40, GITR [glucocorticoid-induced tumor necrosis factor receptor family-related protein], 4-1BB) n'est pas affectée. Seuls les ARNm codant ces molécules inhibitrices sont transportés par les microtubules et le moteur moléculaire Kinésine-l pour fusionner avec des complexes ribo-nucléoprotéiques au niveau des granules de stress et, in fine, être traduits en protéines par les ribosomes. Les drogues déstabilisant les microtubules (comme la colchicine) sont bien plus efficaces que celles conduisant à leur stabilisation. Cette étude a mis néanmoins en garde contre l'utilisation de telles drogues chez les patients cancéreux car, au-delà de l'effet bénéfique escompté des thérapies anti-ICP, un co-traitement par des inhibiteurs de microtubules les expose à un risque particulièrement élevé de développer des maladies auto-immunes, comme cela a été observé dans une analyse portant sur 202693 cas d'effets secondaires de patients cancéreux traités par nivolumab, pembrolizumab ou ipilimumab ${ }^{3}$.

\section{Présence de variants solubles de PD-L1}

Plusieurs études ont rapporté la présence d'ICP solubles dans le plasma de patients. Deux mécanismes moléculaires sont à l'origine de ces formes solubles: I'un reposant sur un épissage alternatif aboutissant à l'absence du domaine transmembranaire de la protéine, l'autre faisant intervenir l'action d'ecto-enzymes à la surface cellulaire. La présence de multiples variants de PD-Ll a été rapportée chez des patients atteints d'un cancer

${ }^{3}$ VigiBase de l'Organisation mondiale de la santé (OMS) [13]. 


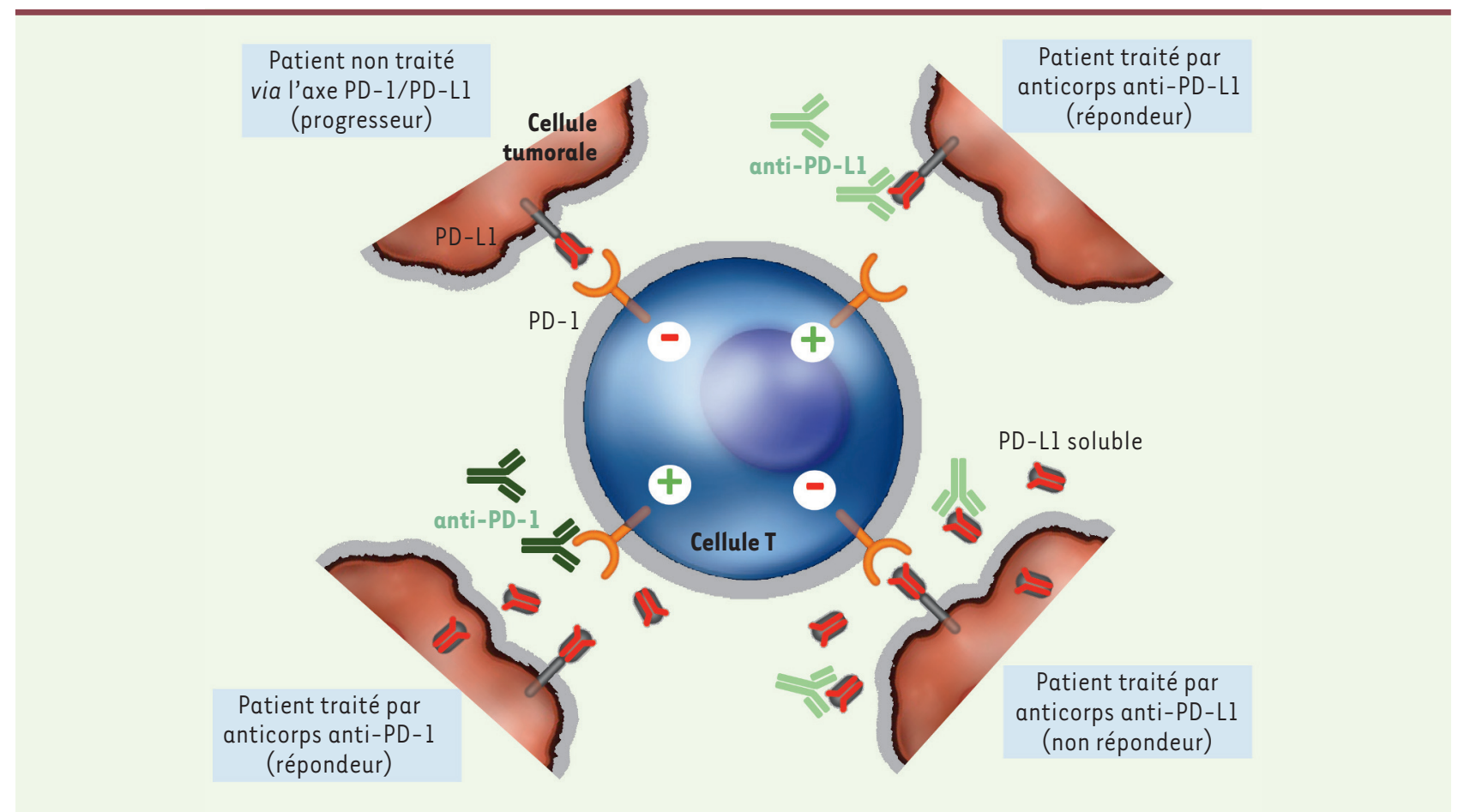

Figure 2. Mécanismes d'action des formes solubles de PD-L1 associées au phénomène de résistance des traitements par anticorps anti-PD-L1.

du poumon et en rechute de leur traitement par anticorps anti-PD-Ll [14]. Des mutations somatiques dans le dernier exon codant le domaine extracellulaire de PD-Ll ont conduit à des formes tronquées dépourvues du domaine transmembranaire. Des mutations ont également été retrouvées dans le gène TDP-43 (TAR DNA-binding protein) dans ces mêmes tumeurs. TDP-43 est un facteur régulant l'épissage alternatif de nombreux gènes et la surexpression in vitro de TDP-43 muté altère le profil des variants de PD-Ll, soulignant l'importance des mécanismes de régulation de la transcription des $A R N$, dans le cancer en particulier. Sur le plan fonctionnel, les deux formes solubles de PD-Ll conservent leur capacité de se fixer à PD-1 (Figure 2) pouvant potentiellement exercer une action inhibitrice à distance de la tumeur. De plus, ces variants solubles sont capables de neutraliser l'anticorps anti-PD-Ll in vivo et, en conséquence, d'abroger l'effet thérapeutique escompté. Une alternative pourrait alors être de traiter les patients par un anticorps anti-PD-1. Cependant, des formes solubles de PD-1 ont été rapportées dans la polyarthrite rhumatoïde. II serait donc judicieux de rechercher la présence de PD-1 soluble avant toute initiation d'une thérapie par anticorps anti-PD-1, et de définir si des formes solubles de PD-1 pourraient être responsables de cas de résistance à ce traitement (voir plus loin). Ces différentes études soulignent donc l'importance de mieux comprendre les éléments de régulation des ICP au niveau transcriptionnel afin d'adapter les traitements combinant des ICP.

\section{Biomarqueurs en immuno-oncologie}

Afin de prédire les chances de réponses ou, a contrario, les risques d'hyper progression ou de toxicité, et de définir les mécanismes de résistance chez les patients non répondeurs à des traitements ciblant PD-1 et CTLA-4, différents biomarqueurs ont été identifiés. II peut s'agir de biomarqueurs immunologiques ou de biomarqueurs génétiques issus de la cellule tumorale et de son microenvironnement [15] ; considérer le biomarqueur en prenant en compte la dynamique de la réponse dans le temps peut également s'avérer particulièrement adapté (Figure 3).

\section{Biomarqueurs immunologiques \\ du microenvironnement tumoral}

\section{Expression de PD-L1}

L'expression de PD-LI sur la cellule tumorale et/ou sur les cellules du microenvironnement tumoral a été l'un des premiers biomarqueurs étudiés, principalement par immuno-histochimie. L'expression de PD-Ll par les cellules tumorales peut se révéler être relativement homogène, comme dans le cancer du poumon, avec des tumeurs complètement négatives et des tumeurs fortement positives [16], ou focale, généralement au contact du stroma, comme dans le cancer de l'ovaire [17]. Les conséquences de cette expression sur le plan clinique sont variables selon le type de cancer. Dans le cancer bronchique non-à-petites cellules (CBNPC), le taux d'expression de $\mathrm{PD}-\mathrm{Ll}$ est associé à la réponse du patient aux thérapies anti-PD-1/PD-L1 [16] : le pembrolizumab (un anticorps anti-PDI) est ainsi approuvé dans le CBNPC sur 


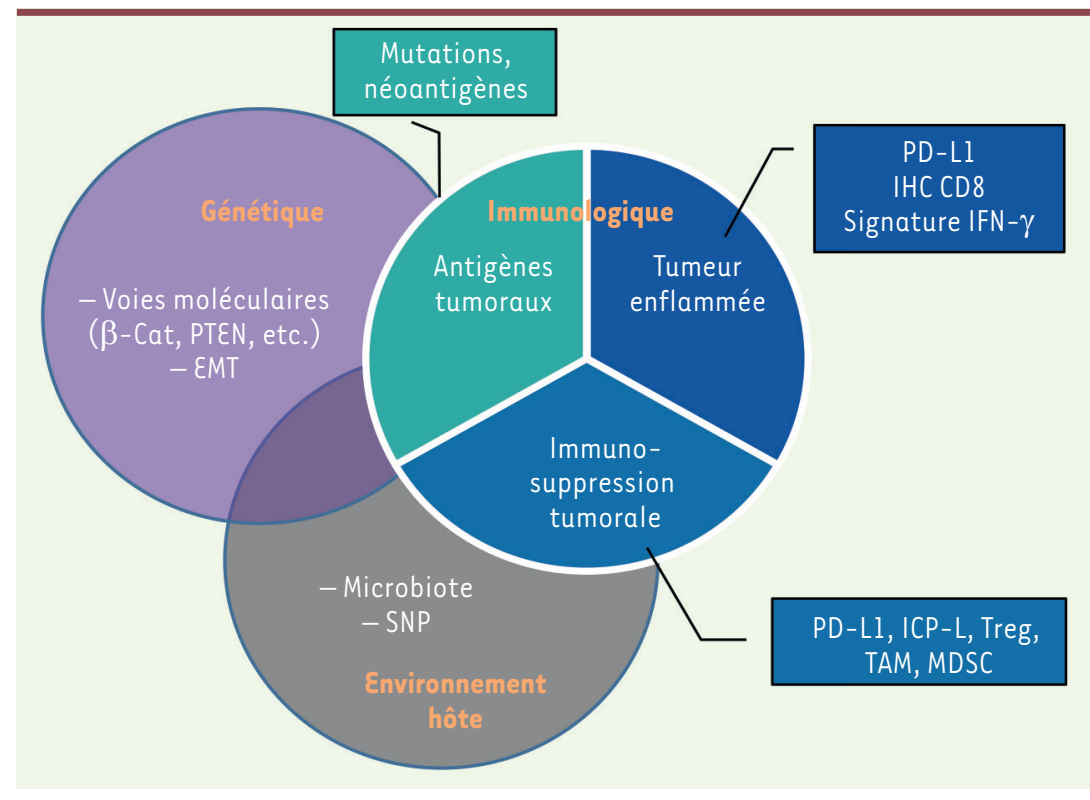

Figure 3. Biomarqueurs tumoraux et immunologiques proposés pour prédire une meilleure réponse à l'immunothérapie.

par les cellules tumorales et par des cellules du microenvironnement, (2) expression focale pouvant conduire à de faux négatifs sur biopsie, (3) expression variable dans le temps et inductible sous immunothérapie, (4) l'impact de son expression peut varier en fonction des combinaisons thérapeutiques et (5) PD-1 peut aussi se lier à PD-L2. Ainsi, pour une meilleure appréciation de l'expression de PD-Ll, y compris au cours du traitement, il sera sans doute nécessaire de développer l'imagerie in vivo [21].

\section{Infiltration par les LT CD8 ${ }^{+}$et signature transcriptomique}

la base de l'expression de PD-Ll par les cellules tumorales. Cependant, dans d'autres types de cancers, comme le carcinome urothélial métastatique, l'expression de PD-LI n'apparaît pas comme étant un marqueur déterminant pour la décision thérapeutique [17].

Il est important de rappeler que l'expression de PD-Ll sur la cellule tumorale peut être induite par les voies oncogéniques, mais surtout en réaction à la réponse immunitaire associée à une production d'IFN (interféron) $-\gamma[18]$ (Figure 4). Cette dynamique d'expression explique probablement que le bénéfice de la combinaison anti-PD- 1 + antiCTLA-4 soit restreint aux patients porteurs d'un mélanome exprimant peu PD-LI [19].

Il est également important de noter que les plus fortes réponses aux anticorps anti-PDI/PD-LI (80-90\%) sont observées dans le lymphome de Hodgkin qui présente une amplification du locus codant PD-L1, PD-L2 et JAK2 (Janus kinase 2) [20].

L'expression de PD-Ll est donc un biomarqueur important, mais son analyse reste complexe et présente plusieurs difficultés : (1) expression

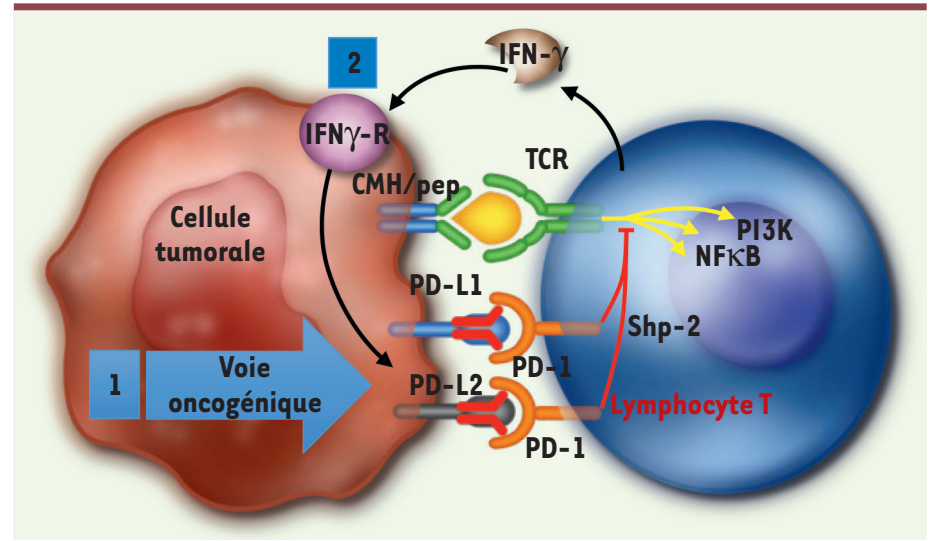

Figure 4. Régulation positive de l'expression de PD-LI induite par l'oncogenèse (1) ou par les lymphocytes T (2) (d'après [18]).
L'infiltration de LT CD8 ${ }^{+}$avant le traitement, qui définit la notion de tumeur chaude vs tumeur froide, est un autre biomarqueur important dont l'impact a été montré dans le mélanome métastatique [22] et dans le cancer du poumon [23]. En complément de leur infiltration, la détection de signatures transcriptomiques des LT activés/enflammés liés à l'IFN- $\gamma$, est associée à une meilleure réponse aux thérapies anti-PD-1/PD-LI dans la plupart des tumeurs solides [24]. En revanche, certains patients dont la tumeur exprime cette signature ne répondent pas à cette immunothérapie, illustrant la nécessité d'identifier des signatures géniques plus spécifiques.

D'autres paramètres immunitaires intra-tumoraux semblent également contribuer à la réponse aux anticorps anti-PDI/LI: 1) l'infiltration des cellules dendritiques et des cellules NK, 2) la co-localisation de PD-1 et de PD-L1, 3) la présence de structures lymphoïdes tertiaires (TLS), plus prédictives que la seule infiltration des LT CD8 ${ }^{+}$[25].

Ces observations plaident pour des études complémentaires afin de connaître l'état d'activation des LT CD $8^{+}$ infiltrants, l'expression d'autres récepteurs co-régulateurs par les $\mathrm{LT} C D 8^{+}$, ainsi que pour l'analyse des $\mathrm{LT}$ $\mathrm{CD} 4^{+}$et d'autres cellules immunitaires.

Biomarqueurs associés à la cellule tumorale : mutations et néo-épitopes

Initialement montrée dans le mélanome et le cancer du poumon [26], puis confirmée récemment dans une étude de phase III dans le cancer du poumon [27], la réponse aux immunothérapies ciblant PD-1 est clairement associée au taux de mutations soma- 


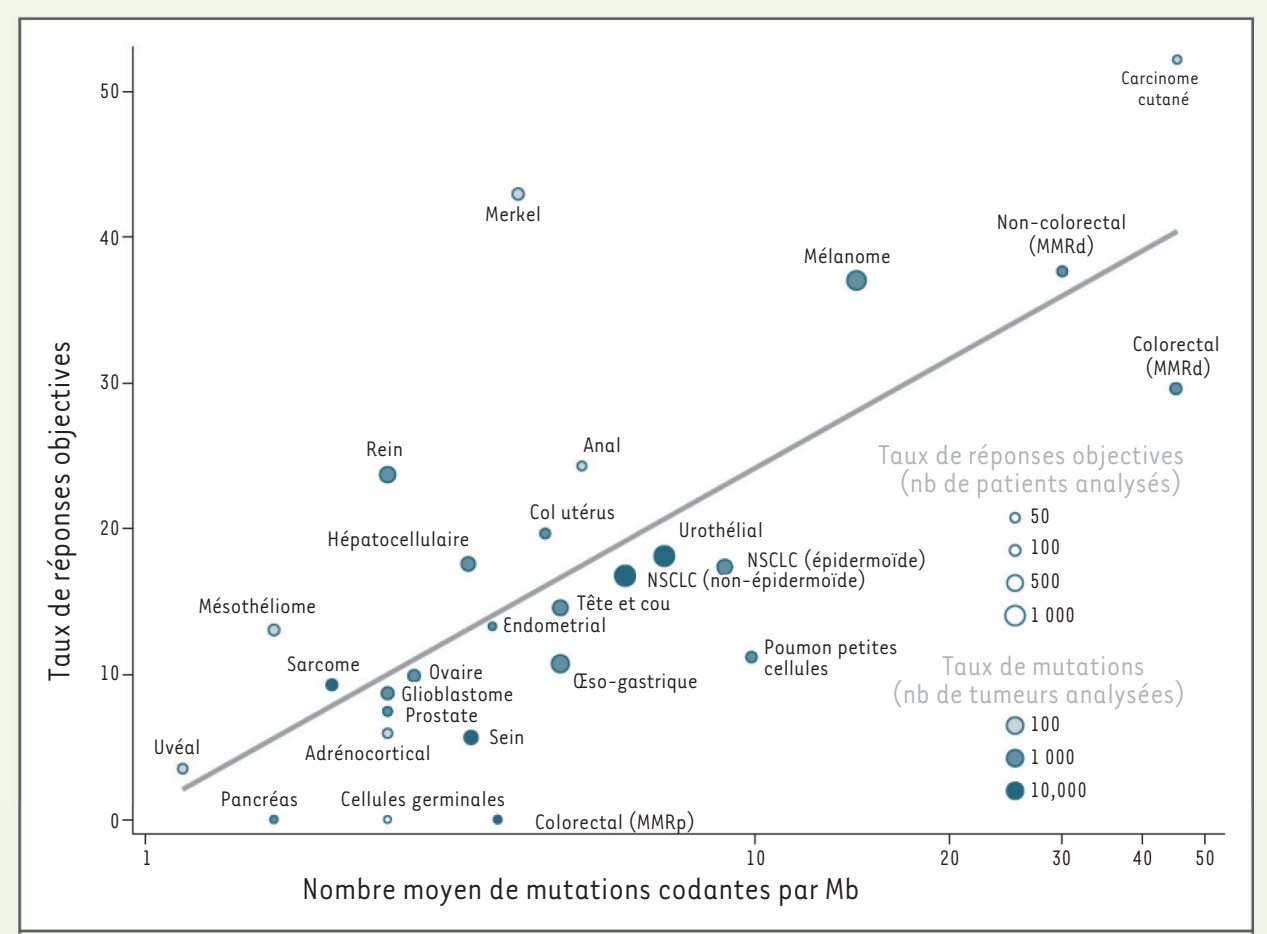

MMRd: mismatch repair deficient MMRp: mismatch repair proficient

Figure 5. Relation entre la charge mutationnelle et la réponse aux immunothérapies ciblant PD-1/PD-LI (d'après [28]).

tiques que présentent les tumeurs, qui peuvent conduire à la génération de néo-épitopes (Figure 5). La plupart des tumeurs ayant un fort taux de mutations somatiques sont en effet associées à de bonnes réponses cliniques au traitement [28]. Des tumeurs porteuses d'instabilités micro-satellitaires (MSI-H) ou d'un défaut de réparation de I'ADN conduisant à l'accumulation de mutations, répondent aux traitements par anticorps anti-PD-1/PD-Ll: le pembrolizumab a ainsi obtenu l'autorisation de mise sur le marché (AMM) pour ces tumeurs non résécables ou métastatiques [29]. II existe cependant des tumeurs présentant un niveau de mutations non négligeable (cas du cancer du côlon non MSI) qui ne répondent pas au traitement par anticorps anti-PDl, suggérant l'existence d'autres voies d'immunosuppression [28]. Le cancer du rein semble également associé à une fréquence élevée d'insertions/ délétions conduisant également à la formation de néo-épitopes [25]. La signature de LT enflammés peut toutefois s'avérer élevée, mais sans corrélation avec le taux de néo-épitopes [30]. Dans leur majorité, les études récentes montrent une bonne réponse des tumeurs d'étiologie virale aux anticorps anti-PD-1/PD-Ll, probablement en lien avec l'expression d'antigènes dominants d'origine virale $[25,31]$ : les tumeurs du col de l'utérus et de la cavité orale liées au papillomavirus (HPV), les tumeurs gastriques liées au virus d'Epstein-Barr ( $\varepsilon B V)$, les carcinomes cutanés de Merkel et les hépatocarcinomes associés au virus de l'hépatite $B$ et $\mathrm{C}(\mathrm{HBV} / \mathrm{HBC})$.
La charge en néo-épitopes apparaît donc associée à la réponse aux anticorps ciblant PD-1. Cependant, une tumeur non infiltrée peut avoir une charge élevée en néo-épitopes et inversement, une tumeur infiltrée, une charge faible. II sera important dans les recherches futures d'inclure l'analyse des néo-épitopes CD8 et CD4 et de leur clonalité, et de l'étendre à d'autres classes d'antigènes tumoraux, y compris d'origine virale.

\section{Prise en compte de la dynamique de la réponse dans le temps}

L'analyse de la dynamique de la réponse au cours du traitement, sur des biopsies tumorales et, d'accès plus aisé, dans le sang périphérique, apporte des informations complémentaires importantes à prendre en considération.

\section{Données sur des biopsies avant et au cours du traitement}

Bien que les informations restent limitées, le niveau des réponses des patients semble associé à une augmentation du nombre de lymphocytes au cours du traitement [22], ainsi qu'à une perte d'expression d'antigènes clonaux [25]. 


\section{Analyses dans le sang}

\section{Cellules immunitaires circulantes}

Un niveau faible de lymphocytes circulants, ou un ratio neutrophiles/ lymphocytes (NLR) élevé avant ou après 2-3 mois de traitement, sont associés à une mauvaise réponse aux anticorps anti-PDl dans l'ensemble des tumeurs solides [32]. Des modifications dynamiques, liées au traitement par anticorps anti-CTLA-4 ou anti-PDl, ont pu être reliées à un bénéfice clinique avec, en particulier : (1) une diminution du nombre de LTreg et de cellules myéloïdes suppressives (MDSC), (2) une augmentation de l'expression d'ICOS par les LT, (3) une fréquence élevée de monocytes activés, et (4) une augmentation de LT CD4 ${ }^{+}$ mémoires [32].

Biomarqueurs immunitaires solubles circulants

Quelques études suggèrent un rôle des cytokines plasmatiques (IFN- $\gamma$, $I L-2$, etc.) dans les effets secondaires, mais pas dans la réponse au traitement [33]. La présence de PD-Ll soluble (sPD-Ll) mais pas de SPD-L2 [34], ainsi que celle d'une forme associée aux exosomes [35], apparaissent de mauvais pronostic dans différentes études. D'autres travaux décrivent un impact négatif des formes solubles de CD73 [36] ou de faibles taux de granzyme B (GranB) soluble [34].

\section{Autres paramètres sanguins}

La diminution à court terme (2-4 semaines) du taux d'IL-8 ou d'ADN tumoral circulants [33], comme marqueurs de la charge tumorale, permettrait de prédire de façon plus précoce que les technologies d'imagerie la réponse clinique à long terme dans le mélanome et le CBNPC. Le taux de $\mathrm{LT} \mathrm{PDI}^{+}$activés circulants est également prédictif d'une réponse anti-tumorale s'il est pondéré par la masse tumorale initiale [37].

Il est donc important de pouvoir réaliser des biopsies précoces sous traitement pour suivre l'évolution de la charge en néo-épitopes et celle de leur clonalité, ainsi que l'évolution du compartiment LT CD8 ${ }^{+}$et de son niveau d'activation. Les analyses réalisées sur le sang peuvent permettre de suivre l'évolution de la masse tumorale et de la réponse immunitaire. II semble donc important de mettre en œuvre des études de suivis dans le sang périphérique de l'ensemble des populations de cellules immunitaires (fréquence, niveau d'activation et fonction) sur de grandes cohortes de patients en vie réelle.

\section{Mécanismes de résistance à l'immunothérapie}

\section{Altération des voies de présentation des antigènes}

Différents mécanismes de résistance, primaire ou acquise, aux immunothérapies commencent à être décrits [31]. La perte d'expression du $\mathrm{CMH}$, par perte d'expression de la $\beta 2$-microglobuline, la perte d'hétérozygotie, ou la régulation négative des ARNm codant les molécules du CMH-I (via MEX3B) ${ }^{4}$ représente un premier mécanisme de résistance.

${ }^{4}$ MEX3B est une protéine qui se lie aux ARNm, qui a été récemment identifiée comme déstabilisant les $\mathrm{ARNm}$ du CMH-l et dont la surexpression est associée à la résistance aux anti-PD-1.
Des mutations ou des délétions dans la voie de I'IFN- $\gamma$ (dont Jak 1/2) ont également été décrites dans plusieurs études. L'inhibition de l'autophagie représenterait également un mécanisme de résistance aux anticorps anti-CTLA-4, mais pas aux anticorps anti-PD-1 via une réduction de la présentation d'antigènes [38].

\section{Autres mécanismes d'immunosuppression dominants}

Les analyses de l'infiltrat immunitaire in situ ou par déconvolution du transcriptome suggèrent que les macrophages et les MDSC s'opposent à la réponse thérapeutique, et même participent au phénomène d'hyperprogression décrit chez certains patients sous traitement par anticorps anti-PD-1 [25].

D'autres récepteurs inhibiteurs exprimés par les LT pourraient également contribuer à la résistance aux anticorps anti-PD-1, tels que LAG-3, TIGIT, VISTA ( $V$-domain Ig suppressor of $T$ cell activation) et plus particulièrement TIM3. Les enzymes CD39 et CD73, de mauvais pronostic dans différents cancers, pourraient également être impliquées, via la production d'adénosine extracellulaire fortement immunosuppressive [25].

\section{Altérations moléculaires intrinsèques à la cellule tumorale}

L'activation de la voie de la $\beta$-caténine dans le mélanome bloque l'infiltration des cellules dendritiques participant au recrutement et à l'activation des LT CD8 ${ }^{+}$. L'inactivation de la voie PTEN (phosphatase and tensin homolog) est, elle aussi, associée à un désert immunitaire et à une absence de réponse à l'immunothérapie dans le mélanome, une observation également confirmée dans les leiomysarcomes utérins ${ }^{5}$ [39]. D'autres altérations moléculaires [de l'EGFR (epidermal growth factor receptor), par exemple], en particulier dans les CBNPC, semblent également s'opposer à la réponse aux anticorps anti-PD-1. Les processus de transition épithélio-mésenchymateuse, de réparation tissulaire, et le caractère souche des cellules tumorales, semblent également associés aux mécanismes de résistance intrinsèque à la cellule tumorale [31].

\section{Stratégies pour lever la résistance aux anticorps anti-PD1}

Ces observations ont conduit au développement de stratégies d'immunothérapie combinant l'injection intra-tumorale de virus oncolytiques et d'anticorps anti-PD-1 permettant une réponse chez plus de $60 \%$ des patients atteints de mélanome métastatique [40].

${ }^{5}$ Le léiomyosarcome utérin est une tumeur maligne, rare, de nature conjonctive, développée aux dépens des éléments mésenchymateux du myomètre. 
De nombreuses autres stratégies sont en cours d'évaluation dans des modèles pré-cliniques ou dans des essais thérapeutiques, telles que la combinaison avec des chimiothérapies immunogènes, des combinaisons d'anticorps anti-ICP (anti-CTLA-4 + anti-PD-1, anti-PD-1 + antiCD73, anti-PD-1 + anticorps agoniste anti-TNFR, etc), l'association à des ligands du TLR3 (Toll-like receptor 3) ou encore l'inhibition des MDSC via la neutralisation du récepteur du fragment C5a du complément (C5aR) [31].

\section{Conclusion}

En conclusion, différentes caractéristiques des ICP, allant de leur rôle régulateur de la réponse immunitaire à leur rôle de cibles pour le développement de médicaments pour les patients cancéreux, doivent être soulignées: 1. L'activation du TCR conduit à la mise en place de freins immunitaires, dont le ciblage a donné des résultats sans précédent quant à la survie sans récidive de certains patients. 2. L'interaction croisée entre récepteurs activateurs et inhibiteurs partageant pour certains les mêmes ligands rend l'équation complexe à modéliser. 3. Un meilleur ciblage des récepteurs inhibiteurs pourrait venir d'une meilleure compréhension de la régulation transcriptionnelle des gènes les codant. L'expression du ligand PD-Ll représente un biomarqueur important bien qu'imparfait, dont l'analyse est rendue complexe du fait de son expression focale par différentes cellules du microenvironnement tumoral et de sa dynamique d'expression au cours du traitement. Si l'infiltration des LT CD8 $8^{+}$, la clonalité des LT infiltrant la tumeur et la signature transcriptomique des LT activés sont associées à une meilleure réponse à l'immunothérapie, il est néanmoins important de mieux caractériser l'état d'activation et l'expression d'autres récepteurs co-régulateurs des $\mathrm{LT} C D 4^{+}$et $\mathrm{CD} 8^{+}$intra-tumoraux, de rechercher des signatures moléculaires plus spécifiques et d'intégrer l'analyse d'autres acteurs de l'immunité. Si la charge et la clonalité des néoépitopes sont globalement associées à la réponse aux immunothérapies ciblant PD-1, une tumeur non infiltrée peut également avoir une charge élevée en néo-épitopes, ce qui implique de réaliser une analyse de la réponse immunitaire contre ces néo-épitopes et d'inclure d'autres antigènes tumoraux. L'importance de l'évolution dynamique de biomarqueurs potentiels rend nécessaire de disposer de biopsies à des temps précoces après le début du traitement, probablement plus informatives que les seules biopsies à TO, et de réaliser une analyse poussée des paramètres immunitaires sanguins. Si divers paramètres immunologiques, ou associés à la tumeur, s'avèrent des biomarqueurs potentiels de réponse à l'immunothérapie, les travaux doivent tendre vers la définition d'un prédicteur composite intégrant tout ou partie de ces différents paramètres.

Il est important de souligner que des paramètres de l'hôte (polymorphismes génétiques) et de son environnement influencent la réponse aux immunothérapies. Des travaux récents ont par exemple montré que le microbiote intestinal influence la réponse aux anticorps antiPD-1/PD-L1 [25].

II faut aussi mentionner que la plupart des études de biomarqueurs de réponse aux immunothérapies actuellement disponibles n'incluent pas de bras contrôle avec le traitement de référence, rendant parfois difficile la distinction entre marqueurs prédictifs et marqueurs pronostiques. $\diamond$

\section{SUMMARY}

The biology of PDI and CTLA-4 as immunotherapeutic targets and the issue of biomarkers

The identification in the 1990's of the role of CTLA-4 and PD-1, two inhibitory receptors of T lymphocytes, in the control of the anti-tumor immune responses, led to the awarding of the Nobel Prize in Physiology or Medicine in 2018 to Dr. James Allison and Dr. Tasuku Honjo. These inhibitory receptors called immune checkpoints are essential to prevent any deleterious impact of on-going immune responses against pathogens or cancer cells on healthy tissues and, hence, guarantee the integrity of the host. These major discoveries have led James Allison and Tasuku Honjo to develop anti-CTLA-4 and anti$\mathrm{PDl} / \mathrm{L}-1$ antibodies, respectively, in order to switch off these immune "brakes", making it possible to efficiently attack tumor cells. CTLA-4 regulates the amplitude of the early $T$-cell activation and inhibits the activity of CD28, a major activating co-receptor expressed by $T$ cells. PD- 1 is expressed by memory and effector T lymphocytes and is involved in the regulation of chronically activated cells, as observed during inflammatory processes. Immunotherapeutic treatments resulting from these discoveries have now a major place in the arsenal of anti-cancer therapies. This review presents firstly a synthesis of knowledge on CTLA-4, PD-1 and their ligands, their mechanisms of action and regulation and, secondly, an overview of biomarkers that have been associated with clinical response to anti-PD-1/PD-L1 and anti-CTLA-4 antibody therapies. $\diamond$

\section{LIENS D'INTÉRÊT}

Les auteurs déclarent n'avoir aucun lien d'intérêt concernant les données publiées dans cet article.

\section{RÉFÉRENCES}

1. Brunet JF, Denizot F, Luciani MF, et al. A new member of the immunoglobulin superfamily-CTLA-4. Nature $1987 ; 328: 267-70$.

2. Ishida Y, Agata Y, Shibahara K, Honjo T. Induced expression of PD-1, a novel member of the immunoglobulin gene superfamily, upon programmed cell death. EMBO J 1992 ; $11: 3887-95$

3. Leung HT, Bradshaw J, Cleaveland JS, Linsley PS. Cytotoxic T lymphocyteassociated molecule-4, a high-avidity receptor for CD80 and CD86, contains an intracellular localization motif in its cytoplasmic tail. J Biol Chem 1995 ; $270: 25107-14$

4. Valk $\varepsilon$, Leung $R$, Kang $H$, et al. T cell receptor-interacting molecule acts as a chaperone to modulate surface expression of the CTLA-4 coreceptor. Immunity $2006 ; 25: 807-21$.

5. Read S, Greenwald R, Izcue A, et al. Blockade of CTLA-4 on CD4+CD25+ regulatory T cells abrogates their function in vivo. J Immunol $2006 ; 177$ : 4376-83.

6. Wing K, Onishi Y, Prieto-Martin P, et al. CTLA-4 control over Foxp3+ regulatory $T$ cell function. Science $2008 ; 322: 271-5$. 


\section{RÉFÉRENCES}

7. Ren J, Han L, Tang J, et al. Foxpl is critical for the maintenance of regulatory T-cell homeostasis and suppressive function. PLoS Biol 2019; 17 : e3000270.

8. Hui $\varepsilon$, Cheung J, Zhu J, et al. T cell costimulatory receptor CD28 is a primary target for PD-1mediated inhibition. Science 2017 ; 355 : 1428-33.

9. Ahn $\varepsilon$, Araki K, Hashimoto M, et al. Role of PD-1 during effector CD8 T cell differentiation. Proc Natl Acad Sci U S A 2018 ; 115 : 4749-4754.

10. Kamphorst AO, Wieland A, Nasti T, et al. Rescue of exhausted CD8 T cells by PD-1-targeted therapies is CD28-dependent. Science 2017 ; 355 : 1423-7.

11. Haile ST, Dalal SP, Clements V, et al. Soluble CD80 restores T cell activation and overcomes tumor cell programmed death ligand 1-mediated immune suppression. J Immunol 2013 ; 191 : 2829-36.

12. Chihara N, Madi A, Kondo T, et al. Induction and transcriptional regulation of the co-inhibitory gene module in T cells. Nature $2018 ; 558: 454-9$.

13. Franchini DM, Lanvin 0 , Tosolini M, et al. Microtubule-driven stress granule dynamics regulate inhibitory immune checkpoint expression in T cells. Cell Rep 2019; 26 : 94-107.e7.

14. Gong B, Kiyotani K, Sakata S, et al. Secreted PD-Ll variants mediate resistance to PD-LI blockade therapy in non-small cell lung cancer. J Exp Med $2019 ; 216: 982-1000$.

15. Blank CU, Haanen JB, Ribas A, Schumacher TN. Cancer immunology. The cancer immunogram. Science $2016 ; 352: 658-60$

16. Garon $\varepsilon B$, Rizvi NA, Hui R, et al. Keynote-001 investigators. Pembrolizumab for the treatment of non-small-cell lung cancer. $N$ Engl J Med $2015 ; 372$ : 2018-28.

17. Nishino M, Ramaiya NH, Hatabu H, Hodi FS. Monitoring immune-checkpoint blockade: response evaluation and biomarker development. Nat Rev Clin Oncol 2017 ; 14 : 655-68.

18. Pardoll DM. The blockade of immune checkpoints in cancer immunotherapy. Nat Rev Cancer 2012 ; $12: 252-64$.

19. Larkin J, Chiarion-Sileni V, Gonzalez R, et al. Combined nivolumab and ipilimumab or monotherapy in untreated melanoma. N Engl J Med 2015 ; $373: 23-34$

20. Ansell SM, Lesokhin AM, Borrello I, et al. PD-1 blockade with nivolumab in relapsed or refractory Hodgkin's lymphoma. N Engl J Med 2015 ; 372 : $311-9$.

21. Bensch F, van der Veen EL, Lub-de Hooge MN, et al. 89Zr-atezolizumab imaging as a non-invasive approach to assess clinical response to PD-Ll blockade in cancer. Nat Med 2018 ; 24 : 1852-8.

22. Tumeh PC, Harview CL, Yearley JH, et al. PD-1 blockade induces responses by inhibiting adaptive immune resistance. Nature $2014 ; 515$ : 568-71.

23. Hu-Lieskovan S, Lisberg A, Zaretsky JM, et al. Tumor characteristics associated with benefit from pembrolizumab in advanced non-small cell lung cancer. Clin Cancer Res 2019 May 21. pii: clincanres.4275.2018.

24. Ott PA, Bang YJ, Piha-Paul SA, et al. T-cell-inflamed gene-expression profile, programmed death ligand 1 expression, and tumor mutational burden predict efficacy in patients treated with pembrolizumab across 20 cancers: keynote-028. J Clin Oncol 2019 ; 37 : 318-27.

25. Havel JJ, Chowell D, Chan TA. The evolving landscape of biomarkers for checkpoint inhibitor immunotherapy. Nat Rev Cancer 2019 ; 19 : 133-50.
26. McGranahan N, Furness AJ, Rosenthal R, et al. Clonal neoantigens elicit T cell immunoreactivity and sensitivity to immune checkpoint blockade. Science $2016 ; 351: 1463-9$.

27. Hellmann MD, Ciuleanu TE, Pluzanski $A$, et al. Nivolumab plus Ipilimumab in Lung Cancer with a High Tumor Mutational Burden. N Engl J Med 2018 ; 378 2093-104.

28. Yarchoan M, Hopkins A, Jaffee $E M$. Tumor mutational burden and response rate to PD-1 inhibition. N Engl J Med 2017 ; 377 : 2500-01.

29. Prasad V, Kaestner V, Mailankody S. Cancer drugs approved based on biomarkers and not tumor type-FDA approval of pembrolizumab for mismatch repair-deficient solid cancers. JAMA Oncol $2018 ; 4: 157-8$.

30. Spranger S, Gajewski TF. Impact of oncogenic pathways on evasion of antitumour immune responses. Nat Rev Cancer 2018 ; 18 : 139-47.

31. Ribas A, Wolchok JD. Cancer immunotherapy using checkpoint blockade. Science. $2018 ; 359: 1350-5$.

32. Ménétrier-Caux C, Ray-Coquard I, Blay Jy, Caux C. Lymphopenia in cancer patients and its effects on response to immunotherapy: an opportunity fo combination with cytokines? J Immunother Cancer 2019; $7: 85$

33. Yi M, Jiao D, Xu H, et al. Biomarkers for predicting efficacy of PD-1/PD-L1 inhibitors. Mol Cancer 2018; $17: 129$

34. Costantini A, Julie C, Dumenil C, et al. Predictive role of plasmatic biomarkers in advanced non-small cell lung cancer treated by nivolumab. Oncoimmunology $2018 ; 7$ : el 1452581 .

35. Chen G, Huang AC, Zhang W, et al. Exosomal PD-Ll contributes to immunosuppression and is associated with anti-PD-1 response. Nature $2018 ; 560: 382-6$

36. Morello S, Capone M, Sorrentino C, et al. Soluble CD73 as biomarker in patients with metastatic melanoma patients treated with nivolumab. $J$ Transl Med 2017; $15: 244$.

37. Huang $A C$, Postow MA, Orlowski RJ, et al. T-cell invigoration to tumour burden ratio associated with anti-PD-1 response. Nature $2017 ; 545: 60-5$.

38. Shukla SA, Bachireddy P, Schilling B, et al. Cancer-germline antigen expression discriminates clinical outcome to CTLA-4 blockade. Cell 2018 ; $173: 624-33 . e 8$.

39. Spranger S, Gajewski TF. Impact of oncogenic pathways on evasion of antitumour immune responses. Nat Rev Cancer 2018 ; 18 : 139-47.

40. Ribas A, Dummer R, Puzanov I, et al. Oncolytic virotherapy promotes intratumoral $\mathrm{T}$ cell infiltration and improves anti-PD-1 immunotherapy. Cell 2017 ; 170 : 1109-19.e10

\section{TIRÉS À PART}

M.C. Dieu-Nosjean

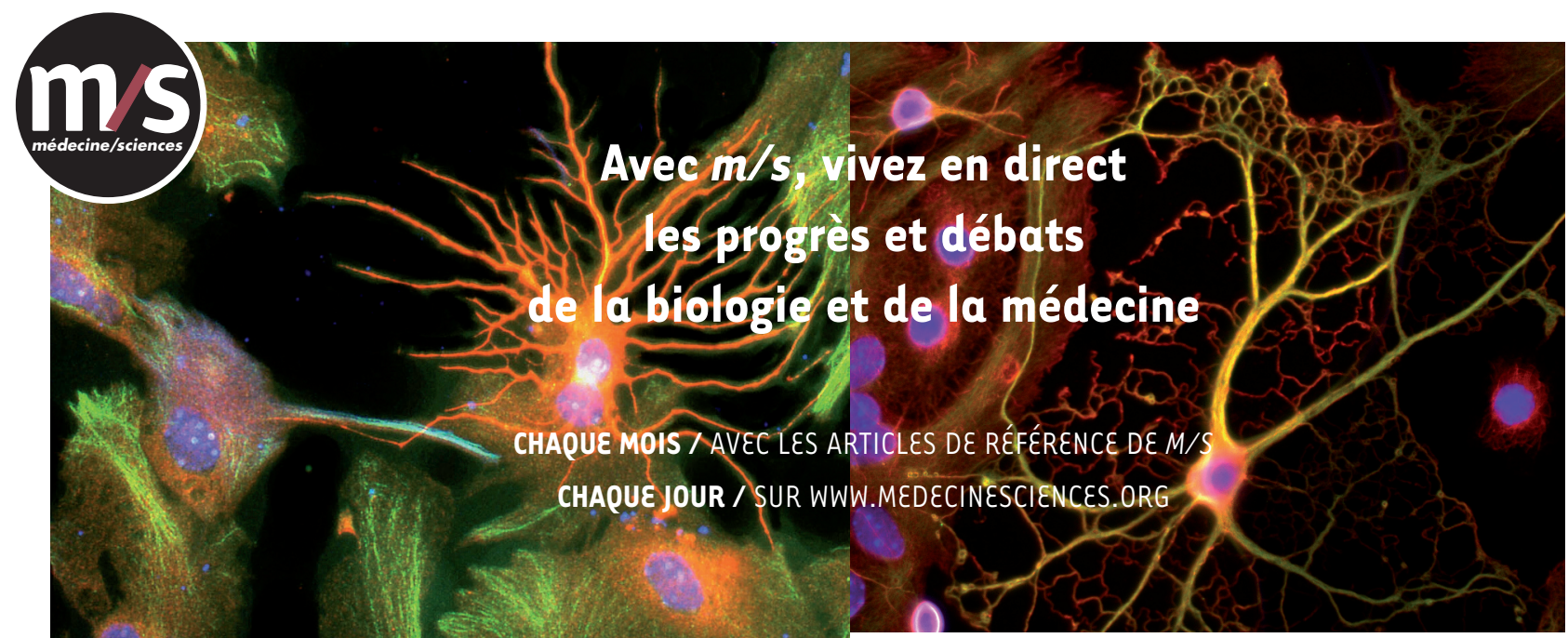

Abonnez-vous sur 